\title{
THE ASTRO-E MISSION
}

\author{
Y. OGAWARA \\ Institute of Space and Astronautical Science, Kanagawa 229
}

\section{Introduction}

The past three decades have seen an explosion in high-energy astrophysics. We have found X-ray astronomy to be an indispensable tool in understanding our Universe. The discipline has become mature, and future X-ray observatories must be more highly specialized. High-resolution spectroscopic imaging in the band above $2 \mathrm{keV}$, systematically exploited by $A S C A$, has led to much new astrophysical knowledge. $A S C A$ has also been playing a particularly important role in studying sources hidden behind dense material. Astro-E, the successor of $A S C A$, is scheduled for launch in the year 2000 by the Institute of Space and Astronautical Science (ISAS) with its newly developed M-V rocket (Ogawara \& Inoue 1997). Our new Astro$E$ observatory features high energy resolution and high sensitivity over the broad energy range $0.5 \mathrm{keV}$ to $600 \mathrm{keV}$. The general emphasis of the observatory is to provide large collecting areas at higher energies, with angular resolution good enough to avoid the confusion limit. This paper provides a brief description of the performance of the Astro- $E$ instruments.

\section{Scientific Instruments}

Astro-E carries two focal-plane instruments for soft X-ray observations, and one collimated large-area counter array for hard X-rays (Table 1). A microcalorimeter array (X-ray Spectrometer - XRS) and four identical sets of $\mathrm{X}$-ray CCDs (X-ray Imaging Spectrometers - XIS) cover the energy band from $0.5 \mathrm{keV}$ to $\sim 10 \mathrm{keV}$ with imaging capability. The Hard X-ray Detector (HXD) is a combination of scintillation detectors and silicon PIN detectors, which cover the hard X-ray band from $10 \mathrm{keV}$ to $600 \mathrm{keV}$. These instruments are being prepared in an extensive collaboration between scientists from Japan and the United States. 
Table 1. Comparison of Astro-E and $A S C A$

\begin{tabular}{|c|c|c|c|}
\hline \multirow[b]{2}{*}{ Focal Plane Detector } & \multicolumn{2}{|c|}{ Astro-E } & \multirow{2}{*}{$\begin{array}{c}A S C A \\
\text { SIS/GIS }\end{array}$} \\
\hline & XRS & XIS & \\
\hline Number of Units & 1 & 4 & $2 / 2$ \\
\hline Focal Length & $4.5 \mathrm{~m}$ & $4.75 \mathrm{~m}$ & $3.5 \mathrm{~m}$ \\
\hline Outer Diameter & $400 \mathrm{~mm}$ & $400 \mathrm{~mm}$ & $345 \mathrm{~mm}$ \\
\hline Number of Layers & 168 & 175 & 120 \\
\hline Surface & Ir or $\mathrm{Pt}$ & $A \mathbf{u}$ & Au \\
\hline Effective Area (1.5 keV) & $\sim 460 \mathrm{~cm}^{2}$ & $\sim 460 \mathrm{~cm}^{2}$ & $\sim 310 \mathrm{~cm}^{2}$ \\
\hline Effective Area $(8.0 \mathrm{keV})$ & $\sim 280 \mathrm{~cm}^{2}$ & $\sim 250 \mathrm{~cm}^{2}$ & $\sim 120 \mathrm{~cm}^{2}$ \\
\hline Half Power Diameter & \multicolumn{2}{|c|}{$1.5^{\prime}$} & $3.5^{\prime}$ \\
\hline Weight / Unit & \multicolumn{2}{|c|}{$20 \mathrm{~kg}$} & $10 \mathrm{~kg}$ \\
\hline
\end{tabular}

Five telescope modules provide flux for the soft X-ray focal-plane instruments. One telescope module with a focal length of $450 \mathrm{~cm}$ feeds the XRS; the other modules have XIS units at their focal planes. The telescopes consist of nested conical thin-foil mirrors based on a similar design concept with the $A S C A$ telescopes (Seremitos et al. 1995). They are made of replica foils and allow a half-power diameter of $\sim 1.5^{\prime}$, which is better than $A S C A$ by at least a factor of two. Additionally, the ratio of the focal length to the diameter is smaller, making the grazing angles smaller. This leads to better reflectivity for higher photon energies. The focal lengths of $4.5 \mathrm{~m}$ for the XRS and $4.75 \mathrm{~m}$ for the XIS give effective areas of $\sim 500 \mathrm{~cm}^{2}$ at $1.5 \mathrm{keV}$ and $\sim 300 \mathrm{~cm}^{2}$ at $8 \mathrm{keV}$. The collecting area for the four XIS units is roughly twice as great as that of $A S C A$.

The microcalorimeter array employed in the XRS is the first such instrument to fly in a satellite and will have an ultimate energy resolution of $12 \mathrm{eV} \mathrm{FWHM} \mathrm{at} 6.4 \mathrm{keV}$ (McCammon et al. 1996). It allows us to perform precise diagnostics of cosmic hot plasmas at a level of physical knowledge never achieved in the past. In the microcalorimeter we take advantage of the low energies of phonons at very low temperature. The small heat capacities at low temperature lead to small fluctuations in the numbers of phonons, resulting in higher energy resolution compared to ordinary semiconductor devices (Moseley et al. 1984). The microcalorimeter must be operated at about $65 \mathrm{mK}$. It will be cooled by an adiabatic-demagnetization refrigerator embedded in a liquid helium/solid neon dewar system. The cryogenic system limits the life of the XRS to about two years (Voltz et al. 1996).

The Astro-E XRS array consists of $32 \mathrm{Si}$ pixels containing ion-implanted thermistors. HgTe with high quantum efficiency through $10 \mathrm{keV}$ is attached 


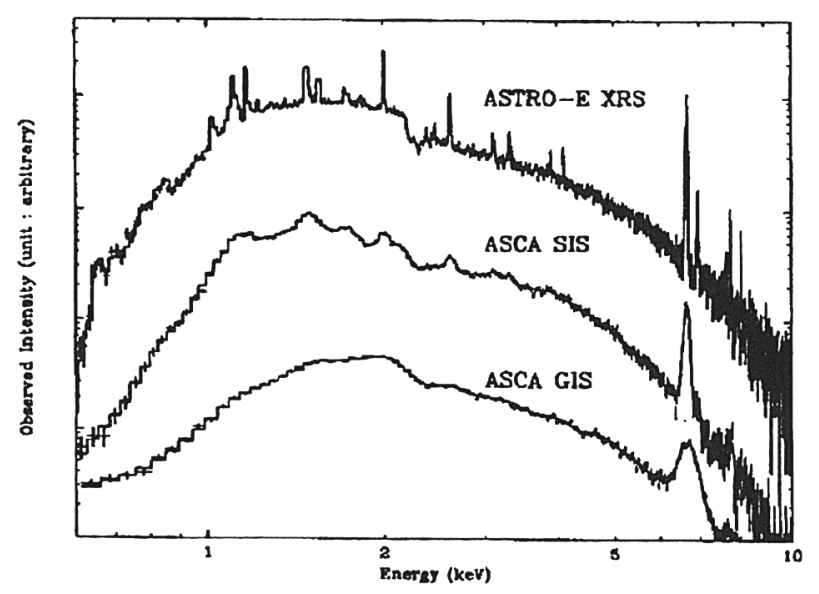

Figure 1. Spectroscopy with the Astro-E microcalorimeter array. The upper curve shows the 12-eV resolution expected from XRS; the lower two curves show the same spectrum at $A S C A$ resolutions.

to the Si pixel as the X-ray absorber (Stahle et al. 1996). The characteristics of the XRS are summarized in Table 2 of Takahashi et al. (1998), and we illustrate in Figure 1 the remarkable improvement in spectral resolution to be achieved. Although the sky coverage is small $\left(2^{\prime} \times 4^{\prime}\right.$ for $2 \times 16$ pixels $)$, we also have imaging capability at a resolution of $\sim 0.5^{\prime}$.

The imaging capability of the Astro-E CCDs (XIS) will play a key role in deep surveys. The SIS units on board $A S C A$ were the first X-ray detectors in space using CCDs in a photon-counting mode. Many efforts have been made to improve the performance of the CCDs and their related electronics, based on $A S C A$ experience. These CCDs, like $A S C A$ 's, are frontilluminated frame-transfer devices with $1024 \times 1024$ pixels, developed by MIT. They have thicker depletion regions than those on $A S C A(\sim 60 \mu \mathrm{m} v \mathrm{~s}$ $\sim 30 \mu \mathrm{m}$ ). A weak radioisotope source at each corner of each chip provides known photon energies for gain calibration. In the XIS, we have the capability to monitor the dark current on a pixel-by-pixel basis, rather than globally as with ASCA. The lower operating temperature, $180-190 \mathrm{~K}$, is also expected to yield better performance in terms of charge transfer efficiency.

Astro- $E$ also carries a Hard X-ray Detector (HXD) array (Takahashi et al. 1996; Kamae et al. 1996), covering the non-thermal energy range $10 \mathrm{keV}$ to $600 \mathrm{keV}$. The well-type phoswich counters (GSO/BGO) provide nearly $4 \pi$ sr shielding (see Table 4 of Takahashi et al., 1998). The detector will thus be characterized by a low background rate, expected to be $\sim 10^{-5}$ photons $/ \mathrm{s} / \mathrm{cm}^{2} / \mathrm{keV}$ at $100 \mathrm{keV}$. It consists of $16(4 \times 4)$ modular units and 
has an overall photon collecting area of about $350 \mathrm{~cm}^{2}$. Two layers of large $\left(2 \times 2 \mathrm{~cm}^{2}\right) 2 \mathrm{~mm}$-thick PIN (silicon) detectors are buried in the deep BGO well just above the detection part of the well-type phoswich counter. Softer $\mathrm{X}$-rays are absorbed in the PIN diodes; harder ones penetrate the diodes and are absorbed by the GSO phoswich. The energy resolution of the HXD is $\sim 4$ $\mathrm{keV}$ below $50 \mathrm{keV}$ (by the silicon detector) and 8-9\% at $662 \mathrm{keV}$. Although the HXD does not have imaging capability, the field of view of the detector for high energy photons is restricted to $4^{\circ} \times 4^{\circ}$ by active collimators made of BGO, and the field of view for low energy photons (below $\sim 100 \mathrm{keV}$ ) is restricted to $0.5^{\circ} \times 0.5^{\circ}$ by fine passive collimators made of phosphor-bronze sheets placed inside the BGO well. The sensitivity of the HXD for point sources should be substantially higher than any other past mission in the energy band between $10 \mathrm{keV}$ to several $100 \mathrm{keV}$. We therefore expect to detect and study many new cosmic hard X-ray point sources.

\section{Conclusions}

With the three experiments described above, the Astro- $E$ mission will be able to perform various kinds of studies for a wide variety of X-ray sources with the highest energy resolution and with the highest sensitivity over a wide energy range from 0.5 to $700 \mathrm{keV}$ ever achieved. Because of the improved image quality and the large effective area, the sensitivity limit by the XIS will improve over $A S C A$ 's and is expected to be $\sim 1 \times 10^{-14} \mathrm{erg} / \mathrm{cm}^{2} / \mathrm{s}$ in the 2-10 keV band. Wide-band spectroscopy with Astro- $E$ should play an important role in studies of the nature of highly-absorbed sources and their contributions to the cosmic X-ray background. The construction of the flight model for Astro- $E$ is underway at the time of writing. The first end-to-end test of the satellite is scheduled in July 1998, leading to a launch in early 2000.

\section{References}

Kamae, T., et al., 1996, SPIE 2806, 315

McCammon D., et al., 1996, NIM, A370, 266

Moseley, S.H., et al., J. Appl. Phys, 56, 1984, 1257

Ogawara, Y., and Inoue, H., 1997, in Proc. 7th ISCOPS Conference (American Astronautical Society, to be published)

Serlemitsos, P., et al., PASJ, 47, 1995, 105

Stahle, C.M., et al., 1996, NIM, A370, 173

Takahasi,T., et al., 1996, A\&AS, 120, 645

Takahasi, T. Inoue, H., \& Ogawara, Y., 1998, Astr. Nachrichten, to be published

Voltz, S.M., et al., 1996, Cryogenics, 36, 763 